MODELING, IDENTIFICATION AND CONTROL, 1993, VOL. 14, NO. 3, 145-159

doi:10.4173/mic.1993.3.3

\title{
Finite element modeling of the hydrodynamic environment of a small ROV
}

\author{
GUANG REN $\ddagger$ and JENS G. BALCHEN $\ddagger$
}

Keywords: Moving boundary, finite element, hydrodynamic model, AUV

This paper addresses a practical problem, namely, modeling the hydrodynamic environment of a small ROV. This has become the problem of solving timedependent incompressible Navier-Stokes equations with moving boundaries and a new method is developed to solve it. Navier-Stokes equations expressed in a moving-body-fixed coordinate frame with moving boundaries are derived and solved by a proposed finite element method which is a modified velocity correction procedure (Ren and Utnes 1993). The present method is implemented in the C language on a SUN/Sparc Station. The algorithm and program are demonstrated by solving a classic driven cavity flow problem and a simplified model of the hydrodynamic environment of a small ROV, which is a moving boundary problem. The results from the driven cavity flow problem are compared to previous work. A definition is also given of the moving boundary problem (MBP) related to the solution of Navier-Stokes equations.

\section{Introduction}

It is important for an autonomous underwater vehicle (AUV) to have both a vehicle model and an environmental model. Many published papers have contributed to the vehicle model (e.g. Yoerger and Slotine 1985, Yoerger, Newman and Sloting 1986, Fossen and Balchen 1988, Cristi, Papoulias and Healey 1990, Goheen and Jefferys 1990, Silvestre, Lemos, Sequeira and Sentiero 1990, Yuh 1990a, 1990b, Fossen 1991, Triantafyllou and Grosenbaugh 1991). However, the literature has scarcely contributed to the hydrodynamic environmental-model related to the control of AUV, which is a significant step to achieve a high degree of autonomous control for such a vehicle. This situation is remedied in the present paper. The task of modeling the hydrodynamic environment turns out to be a moving boundary problem (MBP) which is an interesting but difficult issue. The approach in this paper has been the development of an experimental system (i.e. a small ROV system) and considering how to model the hydrodynamic environment of the small ROV system.

Two kinds of hydrodynamic environmental models are classified. The first is of an AUV moving far away from any structure. In this case, the environment can be modeled as that of a body moving in an infinite domain. This kind of problem can be solved by the methods proposed by, for example, Gresho, Chan, Lee and Upson 1984, Kovacs and Kawahara 1991, Zienkiewicz and Taylor 1991, Ren and Utnes 1992. The second is of an AUV near or inside a structure. The latter problem may be simplified as

Received 16 February 1993.

† On leave from Dalian Maritime University, P.R. China.

$\ddagger$ Department of Engineering Cybernetics, Norwegian Institute of Technology, N-7034 Trondheim, Norway. 


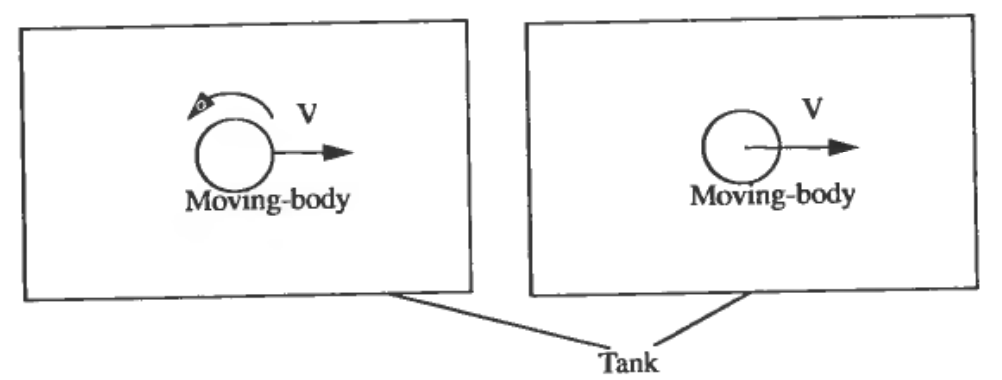

Figure 1. A moving-body in a fixed tank.

that of a body with a thrust moving in a tank, as shown in Fig. 1, which is a moving boundary problem.

There are many methods to solve Navier-Stokes equations with fixed boundary conditions as those mentioned above. However, only relatively few authors have concerned themselves with the solution of Navier-Stokes equations with moving boundaries. Harlow and Welch (1965) developed the marker and cell method to deal with the free surface problem based on a finite difference approach. Viecelli (1971) used a modified version of the marker and cell procedure to solve the same free surface and 'flexible bag' problems. Ogawa and Ishiguro (1987) derived the Navier-Stokes equations expressed in general moving coordinates by using the concept of the Lie derivatives and solved the equations by a finite difference method. Ramaswamy and Kawahara (1987) developed a Lagrangian finite element procedure to solve a viscous free surface fluid flow problem. Demirdzic and Peric (1990) used a finite volume method to solve flow in a channel with a moving indentation problem. Hayashi, Hatanaka and Kawahara (1991) applied a Lagrangian finite element method to solve free surface problems. Tezduyar, Behr and Liou (1992) developed a new strategy using the deforming-spatial-domain/space-time procedure to solve moving boundary problems. However, most of the solution methods presented in the cited references are only applicable to a certain class of problems.

We propose a new method that will solve the Navier-Stokes equations with moving boundaries in a more general fashion. We express and solve the Navier-Stokes equations in a moving-body-fixed coordinate frame. Therefore, we can solve the problem which includes a moving body with a complex shape. Since the most interesting area for our problem in the computational domain is around the moving body, we use a fixed grid in the area near the moving body and an adaptive grid in the area far away from the moving body. A more accurate solution around the moving body can be obtained by this treatment, because no interpolation error is introduced in this area. The present method is applicable to general problems of solving the NavierStokes equations with moving boundaries.

It should be pointed out that the original problem is in three dimensions, however, due to computing power limitations, we only solve the problem in two dimensions.

\section{Definition}

A general definition of MBP can be found in Cryer (1978) and Crank (1984). The definition given here is related to a solution of the time-dependent incompressible viscous Navier-Stokes equations. 


\section{Definition}

A problem described by the time-dependent Navier-Stokes equations defined on a domain $\Omega$, if the domain $\Omega$ is a non-trivial function of time, i.e. $(d / d t) \Omega(t) \neq 0$, then the problem is a moving boundary problem.

\section{Remark}

The driven cavity flow problem is not an MBP although the upper boundary has velocity, because the domain of the problem is fixed. The simplified environmental model of the small ROV is an MBP, according to the definition.

\section{Navier-Stokes equations in a body-fixed coordinate frame}

Newton's law of motion for a fluid continuum takes the form

$$
\rho \frac{d \mathbf{u}}{d t}=-\nabla p+F(\mathbf{u})+\rho \mathbf{g}
$$

where $\mathbf{u}$ is velocity, $p$ is pressure, $\mathbf{g}$ is gravitational acceleration and $F(\mathbf{u})$ is the force. For Newtonian fluid, like air or water (see Pedlosky, 1987)

$$
F(\mathbf{u})=\mu \nabla^{2} \mathbf{u}+\frac{\mu}{3} \nabla(\nabla \cdot \mathbf{u})
$$

where $\mu$ is the molecular viscosity. It is well known that Newton's law applies to the inertial frame. For our applications, we assume that the earth is fixed by a star in space. Now let $\mathbf{r}$ be the position vector of an arbitrary fluid element as shown in Fig. 2. We derive $\mathbf{r}$ with respect to time and get the velocity as:

$$
\left(\frac{d \mathbf{r}}{d t}\right)_{e}=\left(\frac{d \mathbf{r}_{o}}{d t}\right)_{e}+\left(\frac{d \mathbf{r}_{b}}{d t}\right)_{b}+\omega_{b} \times \mathbf{r}_{b}
$$

where the subscripts $e$ and $b$ denote that the derivatives are carried out in the earthfixed coordinate frame and body-fixed coordinate frame, respectively. The velocity $\mathbf{u}_{e}$ $=(d \mathbf{r} / d t)_{e}$ is equal to the sum of $\mathbf{u}_{0 e}=d \mathbf{r}_{0} / d t$ the velocity of the origin of the body-fixed

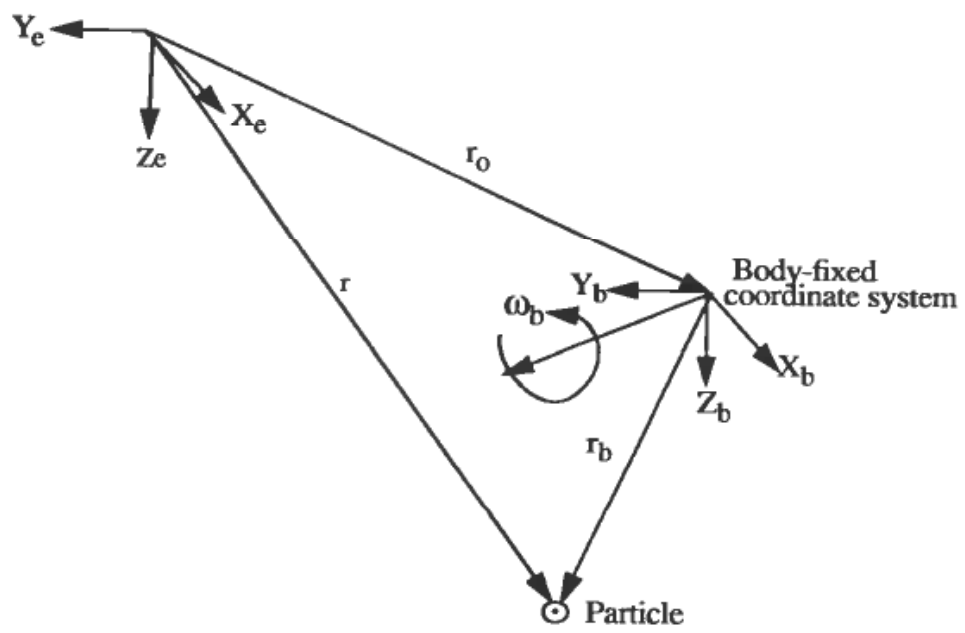

Figure 2. The earth-fixed coordinate system $\mathrm{X}_{\mathrm{e}} \mathrm{Y}_{\mathrm{e}} \mathrm{X}_{\mathrm{e}}$ and the body-fixed coordinate system $\mathbf{X}_{\mathbf{b}} \mathbf{Y}_{\mathbf{b}} \mathbf{X}_{\mathbf{b}}$ 
frame observed in the earth-fixed frame $\mathbf{u}_{b}=d \mathbf{r}_{b} / d t$ the velocity observed in body-fixed frame, and $\omega_{b} \times \mathbf{r}$ the velocity due the rotation. We may write this as

$$
\mathbf{u}_{e}=\mathbf{u}_{o e}+\mathbf{u}_{b}+\omega_{b} \times \mathbf{r}_{b}
$$

Since $u_{o e}$ is the velocity of the origin of the body-fixed coordinates expressed in the earth-fixed coordinate frame, we should transform $\mathbf{u}_{o e}$ from the earth-fixed frame to the body-fixed frame by

$$
\mathbf{u}_{o e}\left(x_{e}, y_{e}, z_{e}\right)=\mathbf{J}_{1}(\phi, \theta, \psi) \mathbf{u}_{o b}\left(x_{b}, y_{b}, z_{b}\right)
$$

where

$$
\mathbf{J}_{1}(\phi, \theta, \psi)=\left[\begin{array}{ccc}
\mathrm{c} \psi \mathrm{c} \theta & \mathrm{c} \psi \mathrm{s} \theta \mathrm{s} \phi-\mathrm{s} \psi \mathrm{c} \phi & \mathrm{c} \psi \mathrm{s} \theta c \phi+s \psi s \phi \\
s \psi c \theta & s \psi \mathrm{s} \theta s \phi+c \psi c \phi & s \psi \mathrm{s} \theta c \phi-c \psi s \phi \\
-s \theta & c \theta s \phi & c \theta c \phi
\end{array}\right]
$$

is the transformation matrix through the functions of Euler angles (see Craig 1989): roll $(\phi)$, pitch $(\theta)$, and yaw $(\psi), s \psi=\sin (\psi), c \psi=\cos (\psi) . \mathbf{J}_{1}$ has a property of $\mathbf{J}_{1} \mathbf{J}_{1}^{\mathrm{T}}$ $=\mathbf{J}_{1} \mathbf{J}_{1}^{-1}=\mathbf{I}$.

Deriving Eqn (4) with respect to time in the body-fixed coordinates, we get

$$
\left(\frac{d \mathbf{u}}{d t}\right)_{e}=\frac{d \mathbf{u}_{o b}}{d t}+\frac{d \mathbf{u}_{b}}{d t}+2 \omega_{b} \times \mathbf{u}_{b}+\omega_{b} \times\left(\omega_{b} \times \mathbf{r}_{b}\right)+\frac{d \omega_{b}}{d t} \times \mathbf{r}_{b}+\omega_{b} \times \mathbf{u}_{o b}
$$

Substituting Eqn (6) into Eqn (1), we have

$$
\begin{aligned}
\rho\left(\frac{d \mathbf{u}_{o b}}{d t}+\frac{d \mathbf{u}_{b}}{d t}+2 \omega_{b} \times \mathbf{u}_{b}+\omega_{b} \times\left(\omega_{b} \times \mathbf{r}_{b}\right)+\frac{d \omega_{b}}{d t} \times \mathbf{r}_{b}+\omega_{b} \times \mathbf{u}_{o b}\right) . \\
=-\nabla p+F\left(\mathbf{u}_{b}\right)+\rho \mathbf{g}
\end{aligned}
$$

For a Newtonian fluid, it is a simple matter to show that (see Pedlosky 1987)

$$
F(\mathbf{u})=F\left(\mathbf{u}_{b}\right)
$$

and $p$ and $\mathbf{g}$ are independent of the coordinate frame. Equation (7) may be written in a conventional form as:

$$
\begin{gathered}
\frac{\partial \mathbf{u}_{b}}{\partial t}+\left(\mathbf{u}_{b} \cdot \nabla\right) \mathbf{u}_{b}+\frac{\nabla p}{\rho}-v \nabla^{2} \mathbf{u}_{b}-2 \omega_{b} \times \mathbf{u}_{b} \\
=-\left(\frac{d \mathbf{u}_{o b}}{d t}+\omega_{b} \times\left(\omega_{b} \times \mathbf{r}_{b}\right)+\frac{d \omega_{b}}{d t} \times \mathbf{r}_{b}+\omega_{b} \times \mathbf{u}_{o b}\right)+\mathbf{g}
\end{gathered}
$$

and the continuity equation

$$
\nabla \cdot \mathbf{u}_{b}=0
$$

where the terms on the right-hand side of Eqn (9) are known source terms or known functions, $\mathbf{u}_{b}$ denotes velocity $\left(\mathrm{ms}^{-1}\right)$ relating to the body-fixed coordinate frame, $v$ is the kinematic viscosity $\left(\mathrm{m}^{2} \mathrm{~s}^{-1}\right)$. The boundary conditions are

$$
\mathbf{u}_{b}=\hat{\mathbf{u}}_{b}(x, t) \text { on } \Gamma(t) \text {, with } \int_{\Gamma} \mathbf{n} \cdot \hat{\mathbf{u}}_{b} d \Gamma=0,
$$


where $\hat{\mathbf{u}}_{b}\left(\mathbf{x}_{b}, t\right)$ is a known function on the boundary $\Gamma(t)$ of the domain $\Omega(t)$ expressed in the body-fixed coordinate frame, and $\mathbf{n}$ is the outward-pointing normal vector on $\Gamma(t)$. The initial conditions are

$$
\begin{aligned}
& \mathbf{u}_{b}=\mathbf{u}_{b}\left(\mathbf{x}, t_{0}\right)=\mathbf{u}_{b}^{0}(\mathbf{x}) \text { in } \Omega\left(t_{0}\right) \cup \Gamma\left(t_{0}\right) \\
& \text { with } \nabla \cdot u_{b}^{0}=0 \text { in } \Omega\left(t_{0}\right) \cup \Gamma\left(t_{0}\right)
\end{aligned}
$$

where $\mathbf{u}_{b}^{0}(\mathbf{x})$ is a prescribed function.

Equation (9) including continuity Eqn (10), are the time-dependent incompressible viscous Navier-Stokes equations in a moving-body-fixed coordinate frame. In the case of $\omega_{b}=0$ and $\mathbf{u}_{o b}=0$, Eqns (9) and (10) are reduced to ordinary Navier-Stokes equations.

\section{Algorithm}

Applying the modified velocity correction method described in detail by Ren and Utnes (1993) to the moving boundary problem we have the following algorithm:

Step 1

Calculation of Runge--Kutta coefficients for an intermediate-velocity $\tilde{u}$ omitting the pressure $\left(\nabla p^{n}\right)$ in Eqn (9):

$$
\mathbf{K}_{\mathbf{1}}=-\Delta t\left(-v \nabla^{2} \mathbf{u}_{b}^{n}+\left(\mathbf{u}_{b}^{n} \cdot \nabla\right) \mathbf{u}_{b}^{n}+\mathbf{S}_{\mathrm{T}}\right)
$$

where $\mathbf{S}_{\mathrm{T}}$ is the source terms $\left(d \mathbf{u}_{o b} / d t+\omega_{b} \times\left(\omega_{b} \times \mathbf{r}_{b}\right)+\left(d \omega_{b} / d t\right) \times \mathbf{r}_{b}+\omega_{b} \times \mathbf{u}_{o b}\right)$ which are supposed known functions.

$$
\begin{gathered}
\hat{\mathbf{u}}=\mathbf{u}_{b}^{n}+\mathbf{K}_{1} \\
\mathbf{K}_{2}=-\Delta t\left(-v \nabla^{2} \hat{\mathbf{u}}+\left(\mathbf{u}^{k} \cdot \nabla\right) \hat{\mathbf{u}}+\mathbf{S}_{\mathrm{T}}\right)
\end{gathered}
$$

where the superscript $n$ indicates the $n$th time step and $\Delta t$ is the time increment.

\section{Step 2}

Calculation of the intermediate-velocity field:

$$
\tilde{\mathbf{u}}=\mathbf{u}_{b}^{n}+\frac{\mathbf{K}_{2}+\mathbf{K}_{1}}{2}
$$

Step 3

Solution of the pressure Poisson equation to satisfy the incompressible continuity equation

$$
\nabla^{2} p^{n}=\left(\frac{\rho}{\Delta t}\right)(\nabla \cdot \tilde{\mathbf{u}})
$$

Step 4

Correction of the intermediate-velocity field:

$$
\mathbf{u}_{b}^{n+1}=\tilde{\mathbf{u}}-\left(\frac{\Delta t}{\rho}\right) \nabla p^{n}
$$




\section{The finite element method}

The finite element discretization of Eqns (13-(18) is performed using the Galerkin weighted residual method through the following expansions in the piecewise polynomial basis functions associated with the finite element method:

$$
\begin{gathered}
u_{b}(\mathbf{x}, t)=\sum_{i=1}^{N} u_{i}(t) \phi_{i}(\mathbf{x}) \\
p(\mathbf{x}, t)=\sum_{i=1}^{N} p_{i}(t) \phi_{i}(\mathbf{x})
\end{gathered}
$$

where $N$ is the number of nodes for velocity and pressure in the discretized domain. The weak form of Eqns (13)-(18) permits $\phi_{i}(\mathbf{x})$ to be discontinuous in the first derivatives and introduces natural boundary conditions. Thus $\phi_{i}(\mathbf{x})$ are chosen to be $C^{0}$ piecewise linear basis functions defined on isoparametric triangular elements. Inserting Eqns (19) and (20) into Eqns (13) (18) leads to a discretized system of equations, which can be written in matrix form for the whole domain.

Step 1

$$
\begin{gathered}
\mathbf{M K}_{\mathbf{1}}=-\Delta t \mathbf{S}^{n} \mathbf{u}_{b}^{n}-\Delta t \mathbf{A}^{n} \mathbf{u}_{b}^{n}+\Delta t \mathbf{M S}_{\mathrm{T}}+\Delta t \Gamma_{u}^{n} \\
\mathbf{M} \hat{\mathbf{u}}=\mathbf{M \mathbf { u } _ { b } ^ { n }}+\mathbf{M K _ { 1 }} \\
\mathbf{M K}_{\mathbf{2}}=-\Delta t \mathbf{S}^{n} \hat{\mathbf{u}}-\Delta t \mathbf{A}^{n} \hat{\mathbf{u}}+\Delta t \mathbf{M S}_{\mathrm{T}}+\Delta t \Gamma_{u}^{n}
\end{gathered}
$$

Step 2

$$
\mathbf{M} \tilde{\mathbf{u}}=\mathbf{M u} \mathbf{u}_{b}^{n}+\left(\frac{\mathbf{M K}_{2}+\mathbf{M K}_{1}}{2}\right)
$$

Step 3

$$
\mathbf{S}_{p} \mathbf{p}^{n}=-\left(\frac{\rho}{\Delta t}\right)\left(\mathbf{D}_{x} \tilde{\mathbf{u}}+\mathbf{D}_{y} \tilde{\mathbf{v}}\right)+\Gamma_{p}^{n}
$$

Step 4

$$
\mathbf{M u} \mathbf{u}^{n+1}=\mathbf{M} \tilde{\mathbf{u}}-\left(\frac{\Delta t}{\rho}\right) \mathbf{D} p^{n}
$$

Here $\mathbf{u}_{b}^{n+1}$ and $\mathbf{u}_{b}^{n}$ are now global vectors containing all nodal values of $\left(u_{b}, v_{b}\right)$ expressed in the body-fixed coordinate frame at the $(n+1)$ th and $n$th time steps, respectively, $\tilde{\mathbf{u}}$ is the global vector for the intermediate velocity field, $p^{n}$ is the global pressure vector containing all nodal values of $p$ (pressure) at the nth time step ( $p$ is defined on the same nodes as $u), \Gamma_{u}^{n}$ and $\Gamma_{p}^{n}$ are the vectors of natural boundary conditions for velocity and pressure respectively, $\mathbf{M}$ is the mass matrix, $\mathbf{S}^{n}$ is the diffusion or Laplacian matrix, and $\mathbf{A}^{n}=\mathbf{A}\left(\mathbf{u}_{b}^{n}\right)$ is the advection matrix.

The element matrices associated with Eqns. (21)-(26) are evaluated on each element as:

$$
\mathbf{M}^{e}=\int_{\mathrm{A}} \Phi \Phi^{\mathrm{T}} d A, \mathbf{S}_{p}^{e}=\int_{\mathrm{A}}\left(\left(\frac{\partial \Phi}{\partial x}, \frac{\partial \Phi}{\partial y}\right)\left[\begin{array}{c}
\frac{\partial \Phi^{\mathrm{T}}}{\partial x} \\
\frac{\partial \Phi^{\mathrm{T}}}{\partial y}
\end{array}\right]\right) d A .
$$


The diffusion matrix $\mathbf{S}^{n}$ has two forms. The original form is

$$
\mathbf{S}^{e n}=\int_{\mathrm{A}}\left(\left(\frac{\partial \Phi}{\partial x}, \frac{\partial \Phi}{\partial y}\right) v\left[\begin{array}{c}
\frac{\partial \Phi^{\mathrm{T}}}{\partial x} \\
\frac{\partial \Phi^{\mathrm{T}}}{\partial y}
\end{array}\right]\right) d A,
$$

and the other version has the balance tensor diffusivity (BTD) included:

$$
\mathbf{S}^{e n}=\int_{\mathrm{A}}\left(\left(\frac{\partial \Phi}{\partial x}, \frac{\partial \Phi}{\partial y}\right)\left[\begin{array}{cc}
v+\frac{\Delta t}{2} \bar{u}_{b} \bar{u}_{b} & \frac{\Delta t}{2} \bar{u}_{b} \bar{v}_{b} \\
\frac{\Delta t}{2} \bar{v}_{b} \bar{u}_{b} & v+\frac{\Delta t}{2} \bar{v}_{b} v_{b}
\end{array}\right]\left[\begin{array}{c}
\frac{\partial \Phi^{\mathrm{T}}}{\partial x} \\
\frac{\partial \Phi^{\mathrm{T}}}{\partial y}
\end{array}\right]\right) d A,
$$

where $\left(\bar{u}_{b}, v_{b}\right)$ are averaged velocities in $x$ and $y$ directions on the element, respectively.

$$
\begin{aligned}
& \mathbf{D}_{x}^{e}=\int_{\mathrm{A}} \Phi\left(\partial \Phi^{\mathrm{T}} / \partial x\right) d A, \\
& \mathbf{D}_{y}^{e}=\int_{\mathrm{A}} \Phi\left(\frac{\partial \Phi^{\mathrm{T}}}{\partial y}\right) d A, D^{e}=\left[\begin{array}{cc}
D_{x}^{e} & 0 \\
0 & D_{y}^{e}
\end{array}\right], A^{e n}=\int_{\mathrm{A}} \Phi\left[u^{n \mathrm{~T}} \Phi\left(\frac{\partial \Phi^{\mathrm{T}}}{\partial x}\right)+v^{n \mathrm{~T}} \Phi\left(\frac{\partial \Phi^{\mathrm{T}}}{\partial x}\right)\right] d A,
\end{aligned}
$$

where $\Phi^{\mathrm{T}}=\left(\phi_{1} \phi_{2}, \phi_{3}\right)$ is the vector of basis functions for an element.

\section{Numerical results}

\subsection{A simplified model of the hydrodynamic environment fo a small ROV}

A simplification of the environmental geometry and boundary conditions of a small ROV is shown in Fig. 3. A circular cylinder (i.e. a small ROV) moves in a rectangular tank. The rectangular tank is reduced to $2 \times 1.2 \mathrm{~m}$, a moving body is a circular cylinder sized $d=0.2 \mathrm{~m}$ in diameter, and it moves in the $x$-direction with velocity $u=0.1 \mathrm{~m} / \mathrm{s}$ and in the $y$-direction with velocity $v=0 \mathrm{~m} / \mathrm{s}$. The Reynolds number is chosen to be

$$
R e=\frac{d u_{b}}{v}=\frac{0.2 \times 0.1}{0.00011428}=175 .
$$

It should be noted that there are two coordinate frames in Fig. 3; the body-fixed and the earth-fixed coordinate frames. Here, the boundary conditions are given in the earthfixed coordinate frame.

The moving body is at a standstill relating to the body-fixed coordinate frame. However, the tank including the fluid is moving relative to the body-fixed coordinate frame. The advantage, here, is that a moving tank is easier to treat than a moving body because: (1) A tank normally has a simple geometric shape. On the other hand, the shape of a moving body may be complex; (2) The most interesting area in the computational domain is the area around the moving body. There will be less computational error in this area, if the computation is done in the moving-body-fixed coordinate frame rather than the earth-fixed coordinate frame, because the grid in this area is fixed with the moving-body, so that no interpolation error is introduced. Figure 4 shows that the subdomains numbered 2,3,4 and 7 are always fixed with moving body. On the other hand, the subdomains numbered 1, 5, 6 and 8 are adapted, which means that the subdomains numbered, 1, 5, 6, 8 are always changing their sizes, when computation is being performed. 


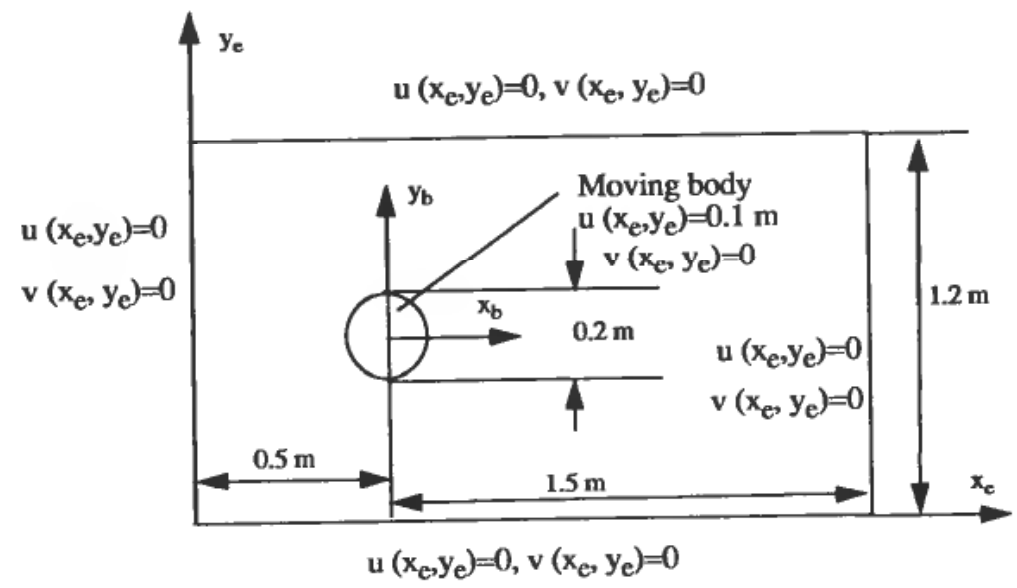

Figure 3. Environmental geometry and boundary conditions.

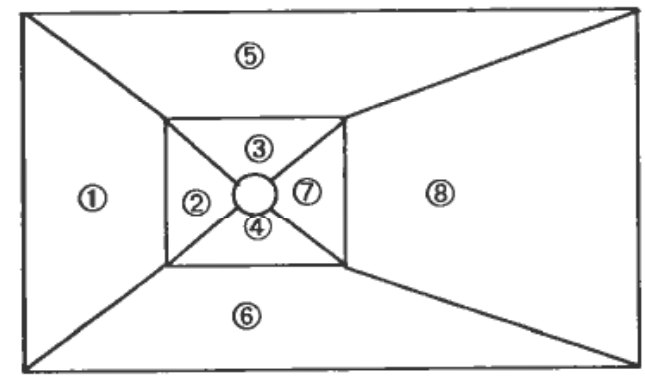

Figure 4. Subdomains.

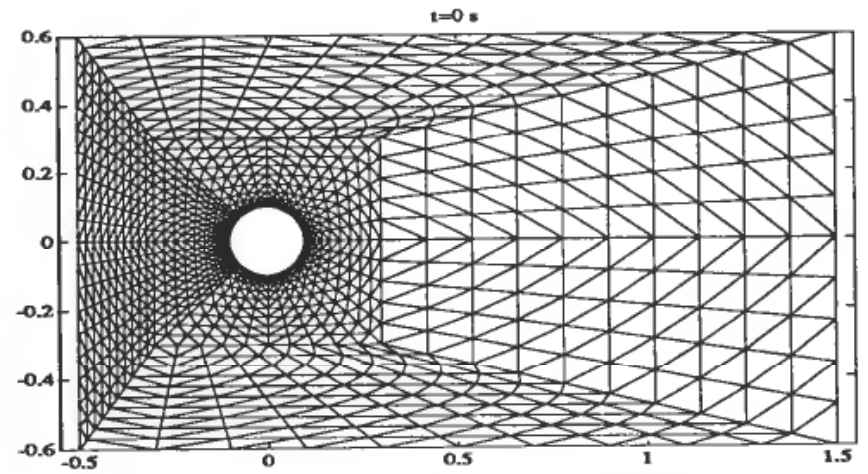

Figure 5. The starting grid.

The grid where the computation starts is shown in Fig. 5. At the same time the body in the tank starts to move with velocity $u=0.1 \mathrm{~m} / \mathrm{s}$ and $v=0 \mathrm{~m} / \mathrm{s}$. A new grid is generated at every time step. The values on the new grid are interpolated from a background grid (the grid before one time step).

The following steps are used for the nodal value $p_{0}$ on the new grid to be interpolated from a background grid in the adapted subdomains. 
Step 1:

Finding nodal point $\mathrm{p}_{\mathrm{op}}$ which is the nearest nodal point on the background grid to nodal point $\mathrm{p}_{\mathrm{o}}$ on the new grid as illustrated in Fig. $6(a)$.

\section{Step 2:}

Finding the nodal points related to point $\mathrm{p}_{\mathrm{op}}$ (the related nodal numbers are stored in an array). Figure $6(b)$ shows the related nodal points to node $p_{o p}$. The nodal points related to nodal point $p_{o p}$ are the points numbered $1,2,3,4,5,6$ and 8 . Point 7 is not related to nodal point $p_{o p}$, because there is no direct link from point $p_{o p}$ to point 7 .

\section{Step 3:}

Evaluating the value of point $p_{0}$ on the new grid using the nodal values of points $p_{1}$, $2,3,4,5,6$ and 8 on the background grid by

$$
V_{0}=\frac{1}{\operatorname{sum}}\left(\frac{1}{\left(d_{0 p}\right)^{2}} V_{o p}+\frac{1}{\left(d_{1}\right)^{2}} V_{1}+\frac{1}{\left(d_{2}\right)^{2}} V_{2}+\ldots+\frac{1}{\left(d_{6}\right)^{2}} V_{6}+\frac{1}{\left(d_{8}\right)^{2}} V_{8}\right)
$$

where $d_{o p}$ denotes the distance from nodal point $\mathrm{p}_{0}$ on the new grid to nodal point $\mathrm{p}_{\mathrm{op}}$ on the background grid, $d_{1}$ is the distance from point $\mathrm{p}_{\mathrm{o}}$ on the new grid to point $\mathrm{p}_{1}$ on the background grid, etc.

$$
\operatorname{sum}=\left(\frac{1}{\left(d_{0 \mathrm{p}}\right)^{2}}+\frac{1}{\left(d_{1}\right)^{2}}+\frac{1}{\left(d_{2}\right)^{2}}+\ldots+\frac{1}{\left(d_{6}\right)^{2}}+\frac{1}{\left(d_{8}\right)^{2}}\right), V_{\mathrm{op}}
$$

is the value (pressure or velocity) on nodal point $\mathrm{p}_{\text {op }}$, and $V_{1}$ is the value (pressure or velocity) on nodal point $p_{1}$, etc.

Figures $7-15$ show the computed results and adapted grids. Figure 7 is the grid at time $t=0.25 \mathrm{~s}$. The cylinder inside the tank is moving in the $x$ direction. Figure 8 shows the velocity field at same instance. It should be noted that the velocity field is expressed in the cylinder-fixed coordinate frame rather than in the earth-fixed coordinate frame and that the movement of the cylinder can be seen from the data on the coordinates. Figure 9 is the grid at time $t=2.5 \mathrm{~s}$. Figure 10 illustrates the velocity field at time $t=2.5 \mathrm{~s}$. Figure 11 is the grid at time $t=5.0 \mathrm{~s}$. Figure 12 is the velocity field at time $t=5.0 \mathrm{~s}$, from which we should note that two eddies are formed behind the cylinder. These results are in good agreement with the experimental data (see Prandtl and Tiejens 1934). Figure 13 illustrates the pressure field at time $t=5 \cdot 0 \mathrm{~s}$. Fig. 14 is the grid at time $t=7.5 \mathrm{~s}$. Figure 15 shows the velocity field at time $t=7.5 \mathrm{~s}$. We note that the cylinder has moved from a position near the left of the tank to a position near the righthand side of the tank.

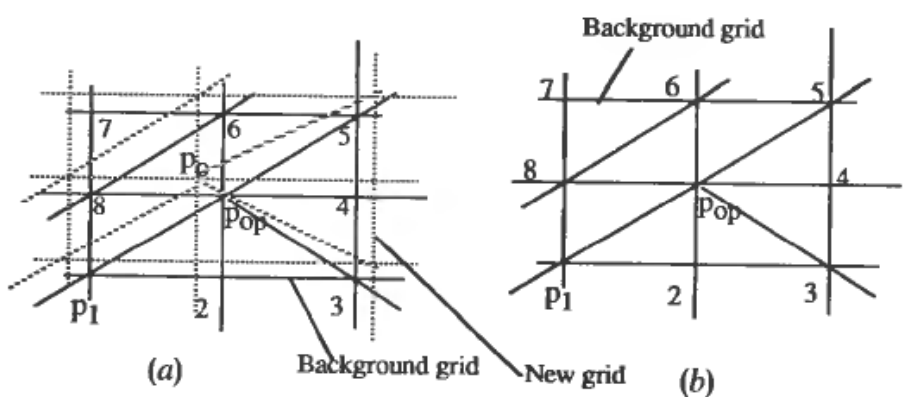

Figure 6. Related nodal points to $\mathrm{pop}_{\mathrm{p}}$. 


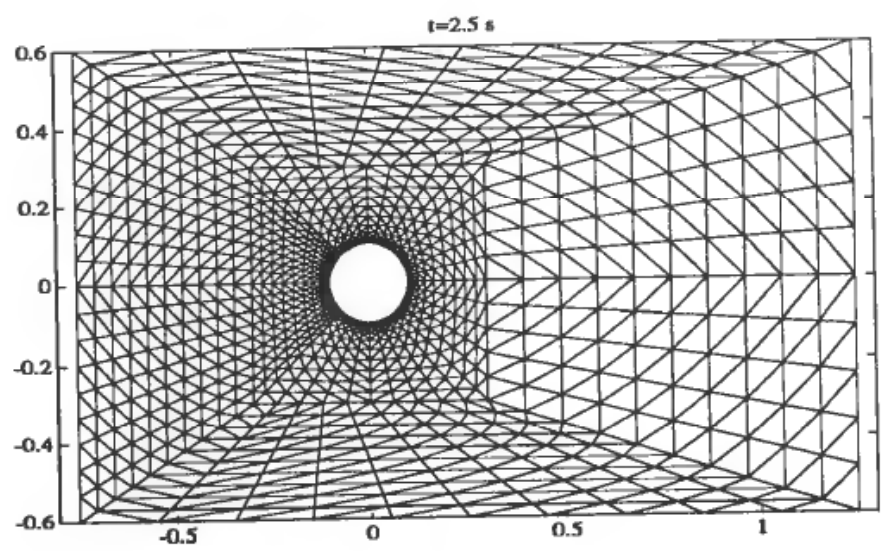

Figure 7. Grid at time $t=0-25, R e=175$.

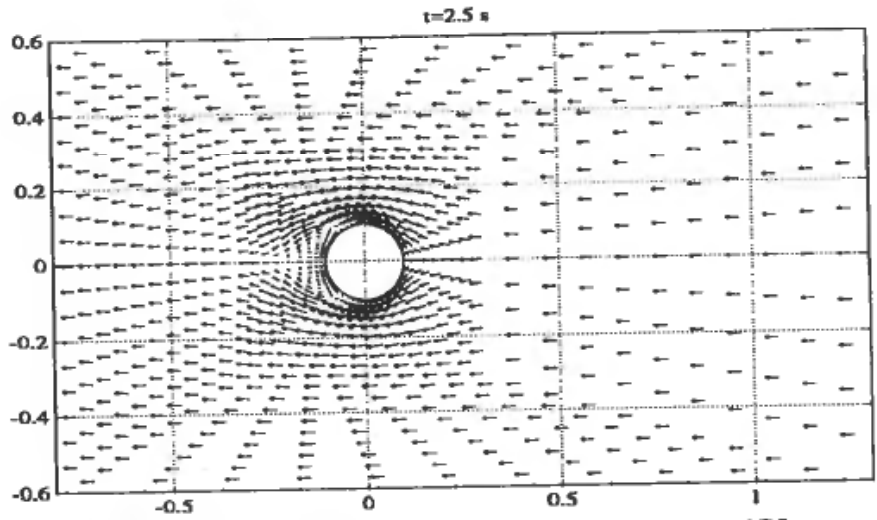

Figure 8. Velocity field at time $t=0 \cdot 25, R e=175$.

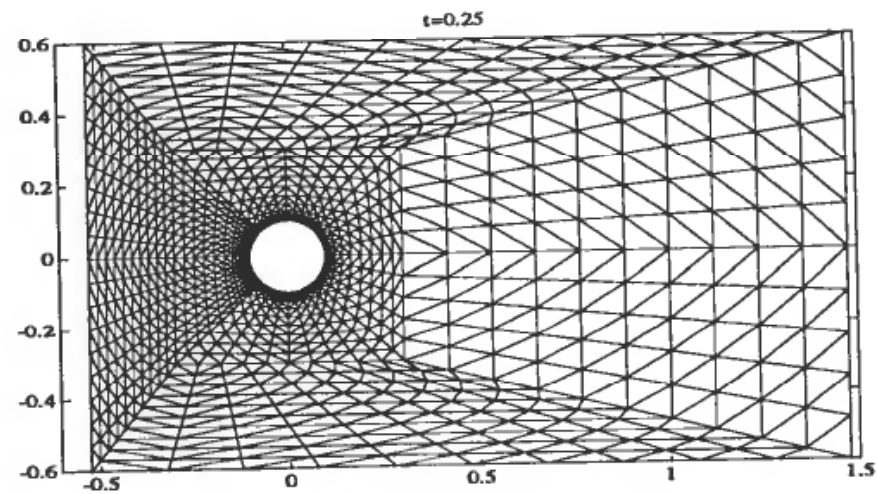

Figure 9. Grid at time $t=2 \cdot 5, R e=175$. 


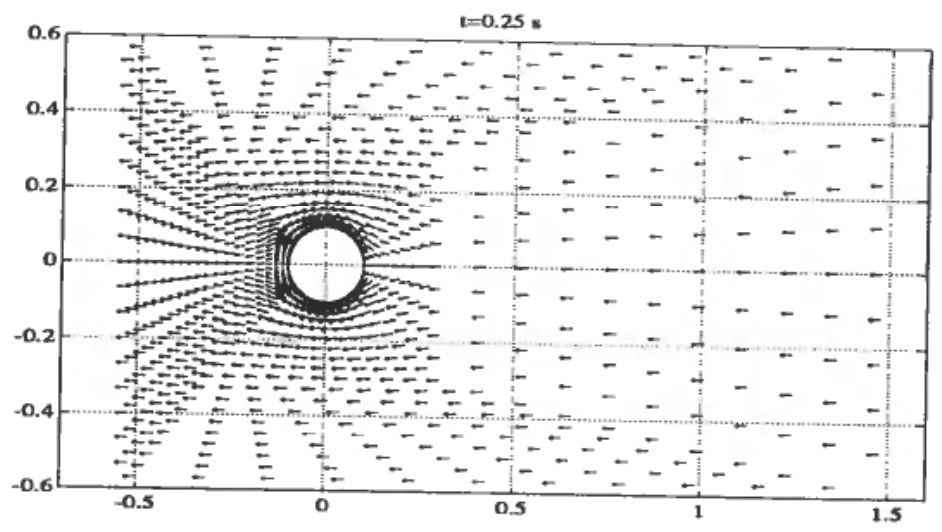

Figure 10. Velocity field at time $t=2.5 \mathrm{~s}, R e=175$.

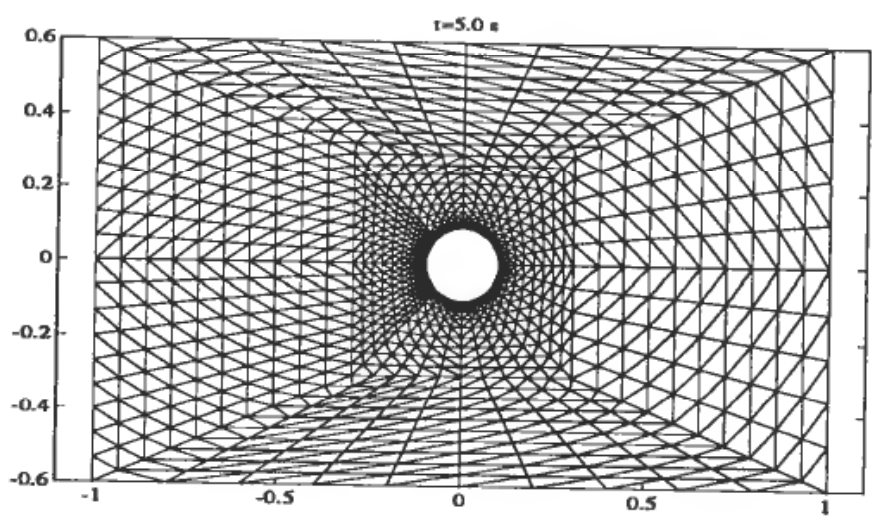

Figure 11. Grid at time $t=5 \cdot 0 \mathrm{~s}, \boldsymbol{R} e=175$.

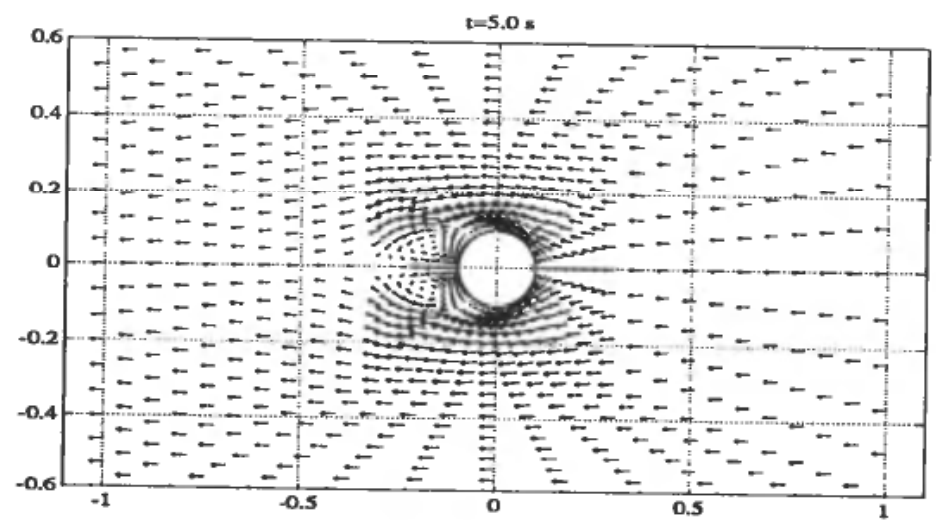

Figure 12. Velocity field at time $t=5 \cdot 0 \mathrm{~s}, R e=175$. 


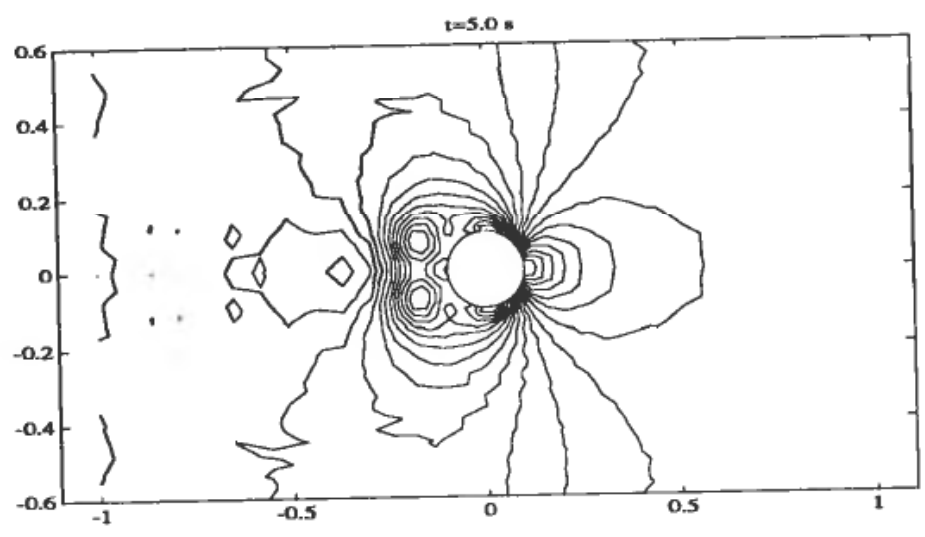

Figure 13. Pressure field at time $t=5 \cdot 0 \mathrm{~s}, \boldsymbol{R e}=175$.

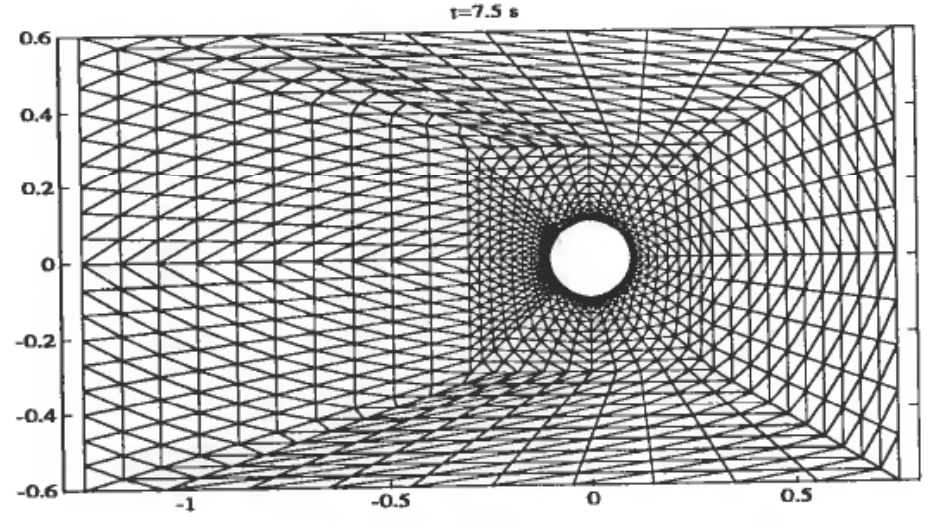

Figure 14. Grid at time $t=7.5 \mathrm{~s}, R e=175$.

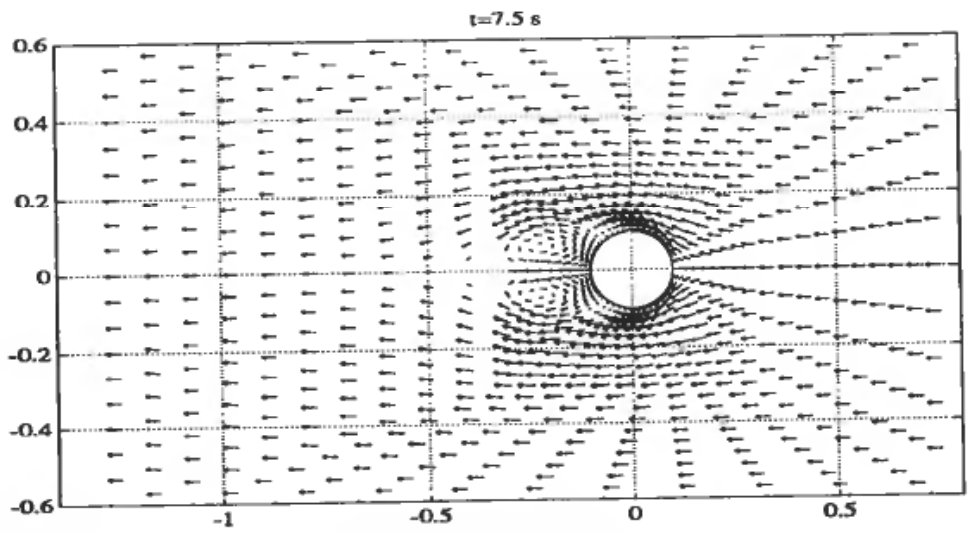

Figure 15. Velocity field at time $t=7 \cdot 5, R e=175$. 


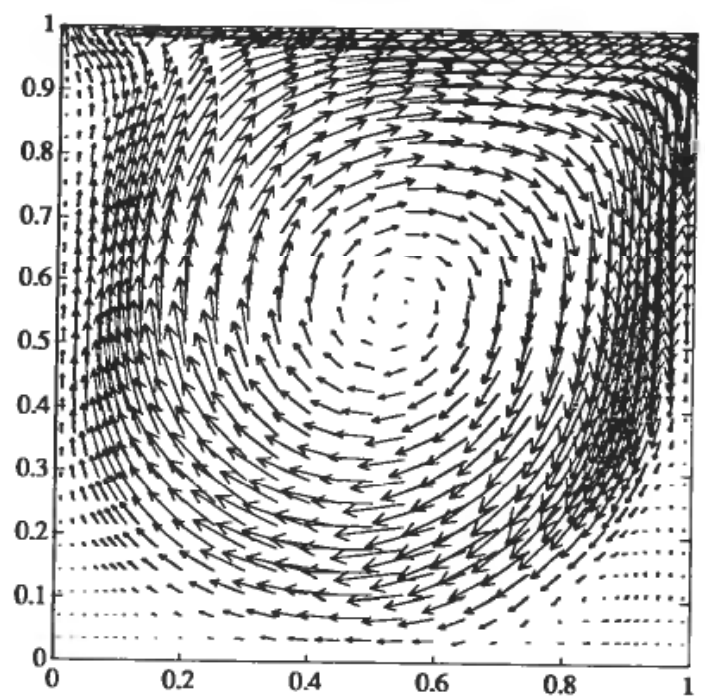

Figure 16. Velocity field of cavity flow, $R e=1000$.

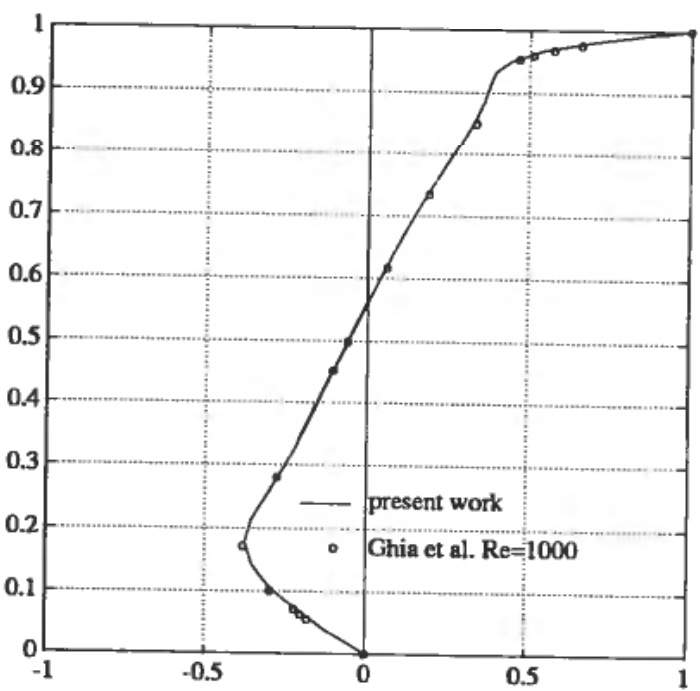

Figure 17. $x$-Velocity distribution through geometric centre of cavity, $R e=1000$.

\subsection{Driven cavity flow}

As mentioned before, in the case of $\omega_{b}=0$ and $\mathbf{u}_{0 b}=0$, Eqns (9) and (10) are reduced to the ordinary Navier-Stokes equations. In order to validate the computing results, we chose the classic driven cavity flow problem as the second example. It seems that the driven cavity flow problem is a standard test and documented data are available for comparison. Reynolds number $\operatorname{Re}=u_{0} L / v=1000$ is selected. A $(32 \times 32)$ grid is chosen, the total number of nodal points are 1089, and there are 2048 elements. The boundary conditions are: A square domain $(1 \times 1 \mathrm{~m})$ with three walls fixed (zero velocity), and the upper wall sliding with constant velocity $u_{0}=1 \mathrm{~m} / \mathrm{s}$. At the two upper corners, $u=0$ and at the first node in from the corners, $u=1 / 2$, as suggested by Sani, Gresho, Lee, Griffiths and Engelman (1981). The time step is $\Delta t=0-01 \mathrm{~s}$. 


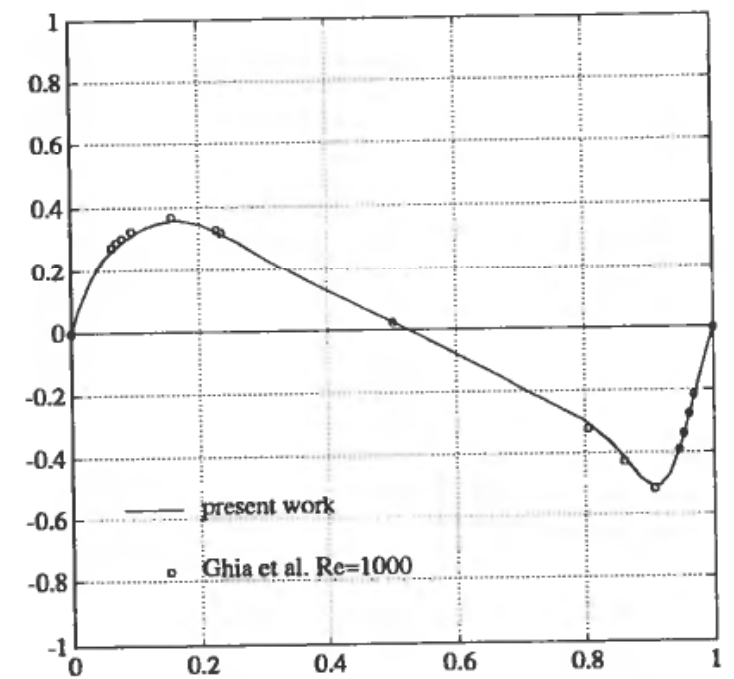

Figure 18. $y$-Velocity distribution through geometric centre of cavity, $R e=1000$.

Figure 16 shows the computed velocity fields. The result compares well with other calculations, e.g. Ghia, Ghia and Shin (1982), as shown in Figs 17 and 18. It should be noted that the present grid is relatively coarse compared to the ones used by Ghia, Ghia and Shin (1982) and Gresho et al. (1984).

\section{Conclusions}

A new method for modeling the hydrodynamic environment of underwater robotic vehicles has been proposed, which is the solution of the time-dependent incompressible Navier-Stokes equations with moving boundaries. The Navier-Stokes equations have been transformed to a general moving-body-fixed coordinate frame. A definition has also been given of the moving boundary problem related to a solution of Navier-Stokes equations. The method presented has been implemented in the $\mathbf{C}$ language on a SUN/Sparc Station, and has been demonstrated by a large number of numerical examples. The code has been validated by comparing the computed results against available experimental data.

Though the purpose of developing this method is to model the hydrodynamic environment of underwater robotic vehicles, it can be seen that this method has a large potential in possible applications to other problems.

\section{ACKNOWLEDGMENTS}

Division of Engineering Cybernetics at Norwegian Institute of Technology provided computing facilities and partially supported this work. The authors are grateful to Dr. Torbjørn Utnes at SINTEF Norwegian Hydrotechnical Laboratory for his kind help.

\section{REFERENCES}

CraiG, J. J. (1989). Introduction to Robotics (Addison-Wesley Publishing Company).

CranK, J. (1984). Free and Moving Boundary Problems (Oxford University Press, London).

Cristi, R., Papoulias, F. A., and Healey, A. J., (1990). Adaptive sliding mode control of autonomous underwater vehicles in the dive plane. I.E.E.E.J. Oceanic Engineering, 15, $152-160$.

CRYER, C. W. (1978). The interrelation between moving boundary problems and free boundary problems. Moving Boundary Problems, D. G. Wilson, A. D. Solomon and P. T. Boggs (ed.) (Academic Press, New York). 
Demirdzic, I. and Peric, M. (1990). Finite volume method for prediction of fluid flow in arbitrarily shaped domains with moving boundaries. Int. J. Numer. Methods Fluids, 10, 771-790.

Fossen, T. I. (1991). Nonlinear Modelling and Control of Underwater Vehicles. Dr. Ing thesis, Norwegian Institute of Technology, Trondheim, Norway.

Fossen, T. I. and BALCHEN, J. G. (1988). Modeling and non-linear self-tuning robust trajectory control of an autonomous underwater vehicle. Modeling, Identification and Control, 9, 165-177.

GHiA, U., GhiA, K. N. and ShIN, C. T. (1982). High-Re solution for incompressible flow using the Navier-Stokes equations and a multigrid method. J. Comput. Phys., 48, 387-411.

GOHEEN, K. R. and Jefferys, R. (1990). Multivariable self-tuning autopilots for autonomous and remotely operated underwater vehicles. I.E.E.E.J. Oceanic Engineering, 15, 144-151.

Gresho, P. M., Chan, S. T., LEE, R. L. and UPSON, C. D. (1984). A modified finite element method for solving the time-dependent incompressible Navier-Stokes equations. Part 2: Applications. Int. J. Numer. Methods Fluid, 4, 619-640.

HaRlow, F. H. and WeLCH, J. E. (1965). Numerical calculation of time-dependent viscous incompressible flow of fluid with free surface. The Physics of Fluids, 8, 2182-2189.

Hayashi, M. HatanaKa, K. and Kawahara, M. (1991). Lagrangian finite element method for free surface Navier-Stokes flow using fraction step methods. Int. J. Numer. Methods Fluids, 13, 805-840.

Kovacs, A. and Kawahara, M. (1991). A finite element scheme based on the velocity correction method for the solution of the time-dependent incompressible Navier-Stokes equations. Int. J. Numer. Methods Fluids, 13, 403-423.

OGAwA, S. and Ishiguro, T. (1987). A method for computing flow fields around moving bodies. J. Comput. Phys., 69, 49-68.

Pedlosky, J. (1987). Geophysical Fluid Dynamics (Springer-Verlag, New York).

PRANDTL, L. and TietJens, O. G. (1934). Applied Hydro- and Aeromechanics (Dover, New York).

Ramaswamy, B. and KaWAHara, M. (1987). Lagrangian finite element analysis applied to viscous free surface fluid flow. Int. J. Numer. Methods Fluids, 7, 953-984.

REN, G. and UTNES, T. (1993). A finite element solution of the time-dependent incompressible Navier-Stokes equations using a modified velocity correction method. Int. J. Numerical Methods in Fluids (to appear).

Sani, R. L., Gresho, P. M., Lee, R. L., Griffiths, D. F. and Engelman, M. (1981). The cause and cure (!) of the spurious pressures generated by certain FEM solutions of the incompressible Navier-Stokes equations: Part 2. Int. J. Numer. Methods Fluids, 1, 171-204.

Silvestre, C., Lemos, J. M., Sequeira, M. M. and Sentieiro, J. S. (1990). Modeling and adaptive control of a deepwater FSV, IF AC 11th Conf., pp. 124-129.

TeZduYAR, T. E. BEHR, M. and LiOU, J. (1992). A new strategy for finite element computations involving moving boundaries and interfaces - the deforming-spatial domain/space-time procedure: I. The concept and the preliminary numerical tests. Computer Methods in Applied Mechanics and Engineering, 94, 339-351.

Triantafyllou, M. S. and Grosenbaugh, M. A., (1991). Robust control for underwater vehicle systems with time delays. I.E.E.E. J. Oceanic Engineering, 16, 146-151.

VIECELLI, J. A. (1971). A computing method for incompressible flows bounded by moving walls. J. Comput. Phys., 8, 119-143.

YOeRGER, D. R. and SLOTINE J.-J. E. (1985). Robust trajectory control of underwater vehicles. I.E.E.E. J. Oceanic Engineering, 10, 462-470.

Yoerger, D. R. Newman, J. B. and Slotine, J.-J. E. (1986). Supervisory control system for the JASON ROV. I.E.E.E. J. Oceanic Engineering, 11, 392-39.

YUH, J. (1990a). A neural net controller for underwater robotic vehicles. I.E.E.E. J. Oceanic Engineering, 15, 161-166.

YUH, J. (1990b). Modeling and control of underwater robotic vehicles. I.E.E.E. Transactions on Systems, Man, and Cybernetics, 20, 1475-1483.

ZiEnKIEWICZ, O. C. and TAYLOR, R. L. (1991). The Finite Element Method. Volume 2 (McGrawHill, London). 